\title{
The use of grape juice in the detection of esophageal leaks
}

\author{
Madison J. Malfitano ${ }^{1}$, Jenny T. Bui ${ }^{2}$, Rachel M. Swier ${ }^{1}$, Benjamin E. Haithcock ${ }^{3}$ \\ ${ }^{1}$ University of North Carolina School of Medicine, Chapel Hill, NC, USA; ${ }^{2}$ Henry Ford Health System, Detroit, MI, USA; ${ }^{3}$ Department of Surgery, \\ Division of Cardiothoracic Surgery, Chapel Hill, NC, USA \\ Contributions: (I) Conception and design: JT Bui, MJ Malfitano, BE Haithcock; (II) Administrative support: JT Bui, MJ Malfitano, BE Haithcock; \\ (III) Provision of study materials or patients: MJ Malfitano, JT Bui, BE Haithcock; (IV) Collection and assembly of data: MJ Malfitano, JT Bui, \\ RM Swier; (V) Data analysis and interpretation: MJ Malfitano, JT Bui, BE Haithcock; (VI) Manuscript writing: All authors; (VII) Final approval of \\ manuscript: All authors. \\ Correspondence to: Benjamin E. Haithcock, MD. The University of North Carolina at Chapel Hill School of Medicine, Burnett-Womack Building, \\ CB \# 7065, Chapel Hill, NC 27599-7065, USA. Email: benjamin_haithcock@med.unc.edu.
}

\begin{abstract}
Background: Esophagectomies and repair of esophageal perforations are operations used for a variety of clinical indications. Anastomotic leaks are a major post-operative complication after these procedures. At our institution, we routinely use grape juice to detect esophageal leaks in the post-operative setting in addition to other standard imaging modalities. We hypothesize that grape juice can provide similar diagnostic sensitivity and specificity to other modalities for leak detection.
\end{abstract}

Methods: A retrospective review of all patients who underwent an esophagectomy or repair of esophageal perforations from 2013-2019 by the thoracic surgery service at our institution was performed. All patients underwent a barium swallow study, CT imaging or upper endoscopy, as well as ingesting purple grape juice on post-operative day 5 or greater. Purple grape juice observed in the tube thoracostomy drainage system was identified as a positive esophageal leak.

Results: Sixty-four patients were included in the study period ( $25 \%$ female, $88 \%$ white, median age 62 years old). Sixty-three patients had both a barium swallow study and grape juice test, while one patient underwent CT imaging and grape juice study. Grape juice test sensitivity and specificity were found to be $80 \%$ and $98.3 \%$, respectively.

Conclusions: This pilot study demonstrates the effectiveness of using grape juice in detecting esophageal leaks after esophageal operations in patients with tube thoracostomies. Grape juice may be cheaper and potentially less morbid than other studies performed to detect esophageal leaks. Further research is needed to justify the increased use of grape juice in patients who undergo esophageal operations.

Keywords: Esophagectomy; esophageal leak; grape juice; barium swallow

Submitted Aug 02, 2021. Accepted for publication Oct 14, 2021.

doi: $10.21037 /$ jtd-21-1185

View this article at: https://dx.doi.org/10.21037/jtd-21-1185

\section{Introduction}

Esophagectomies and esophageal repairs are operations used for a variety of clinical indications ranging from malignancy, traumatic ruptures, and iatrogenic injuries. One of the major complications of esophageal operations postoperatively are anastomotic leaks, likely due to ischemia and mechanical strain (1). Esophageal leaks and ruptures present a serious risk to patient morbidity and mortality with some studies reporting mortality rates as high as $22 \%(2,3)$. Therefore, swift detection is key to initiating appropriate management and limiting morbidity and mortality.

A common standard for detection of leaks following esophageal repair and esophagectomies consists of contrast-mediated barium swallow studies. These are noninvasive techniques that require radiographic still-imaging capabilities and contrast medium. While these are useful in the detection of other esophageal pathologies, they 
may have limited utility in the detection of leaks following surgical repair considering their low sensitivity $(1,4-10)$. In addition, barium swallow studies are associated with adverse complications including nausea, vomiting, inflammatory mediastinitis, pneumonitis, aspiration pneumonia, and rarely, hypersensitivity reactions $(4,11)$.

The standard for esophageal leak detection with postoperative barium swallow study was described by Agha et al. in 1985 using contrast-meditated swallow studies for routine detection of esophageal anastomotic leaks (12). It was previously believed by Tanomkiat and Galassi that barium swallow was a superior technique for investigating the presence of esophageal leaks in the post-operative period, especially when compared to other aqueous contrast studies because it could detect smaller and clinically asymptomatic leaks to a greater degree (13). Hogan et al. described the relatively common nature of leaks following esophagectomy (15-20\%) and noted that surgeons typically rely on a high index of clinical suspicion with common reflex to perform contrast-mediated barium swallow studies (14). However, multiple studies have advised against the use of contrast-mediated swallow studies for both routine post-operative screening and general use when leaks are clinically apparent due to low sensitivities and positive predictive values $(1,4-10)$. Some studies suggest using computed tomography (CT) of the chest or routine post-operative endoscopy for leak detection; however, these have not been widely adopted $(1,4-10)$. There have been concerns regarding the use of endoscopy and its association with increased risk of iatrogenic injury of the anastomoses and gastric conduit (6). Based on Hogan et al.'s study, the use of CT imaging with contrast did not provide substantially added benefit in detection rates compared to barium swallow, but had an added advantage for use in less mobile or neurocognitively impaired patients (14). These findings did not provide enough power for wide adoption of CT based imaging, especially in routine use for leak detection. Therefore, barium swallow studies still are routinely used as a gold standard for detection of anastomotic leaks following esophageal surgery.

The idea to utilize colored liquid for anastomotic leak detection is not uncommon across thoracic and foregut surgeons, however, there have been no reported studies on the effectiveness of routine leak detection with this modality, specifically using purple grape juice. At our institution, in addition to barium swallow studies, we also perform a grape juice swallow study to identify the presence of leaks. Our thoracic surgeons use grape juice in addition to barium swallow studies due to the aforementioned low sensitivity and associated risks of barium swallow.

The goal of this study was to investigate whether a grape juice swallow study is as effective as barium contrast techniques in the routine screening and detection of esophageal leaks following esophagectomies and esophageal repairs. We hypothesize that grape juice can provide similar diagnostic sensitivity and specificity to other modalities for leak detection while also being cheaper and more accessible.

We present the following article in accordance with the STARD reporting checklist (available at https://dx.doi. org/10.21037/jtd-21-1185).

\section{Methods}

\section{Study group}

A retrospective review of all patients who underwent an esophagectomy or repair of esophageal perforations from 2013-2019 by the thoracic surgery service at the University of North Carolina Hospitals was performed. Participants were identified using our Society of Thoracic Surgeons (STS) database. The study was conducted in accordance with the Declaration of Helsinki (as revised in 2013). The study was approved by institutional review board committee (IRB\#: 20-0830) and individualized consent for this retrospective analysis was waived.

\section{Grape juice test procedure}

Patients who underwent an esophagectomy or esophageal repair for an esophageal perforation would have a 28-32 French tube thoracostomy placed intraoperatively. Once the thoracostomy tube is placed on water seal, the grape juice test procedure is as follows: on post-operative day (POD) five or greater, the patient will ingest one, $118 \mathrm{~mL}$ container of purple grape juice. POD 5 is standard for the grape juice and barium swallow study at our institution unless there are clinical indications for a suspected leak sooner. Following immediate ingestion and for up to four hours afterwards, a member of the surgical team will observe for the presence of purple grape juice in the tube thoracostomy drainage system. The detection of purple grape juice is easily detectable even with serosanguinous drainage in the system. If there is no presence of purple grape juice, this is considered a negative test and would suggest the absence of an esophageal leak. If results of the grape juice study are equivocal, then patients receive a barium swallow study 
Table 1 Patient characteristics

\begin{tabular}{|c|c|}
\hline Characteristic & Outcome \\
\hline Total, n & 64 \\
\hline Age, years, median (IQR) & $61.5(16.0)$ \\
\hline \multicolumn{2}{|l|}{ Sex, n (\%) } \\
\hline Male & $48(75.0)$ \\
\hline Female & $16(25.0)$ \\
\hline \multicolumn{2}{|l|}{ Race/ethnicity, n (\%) } \\
\hline Non-Hispanic White & $56(87.5)$ \\
\hline Non-Hispanic Black & $6(9.4)$ \\
\hline Other & $2(3.1)$ \\
\hline \multicolumn{2}{|l|}{ Comorbid conditions, n (\%) } \\
\hline Hypertension & $37(57.8)$ \\
\hline Diabetes & $11(17.2)$ \\
\hline Stroke & $7(10.9)$ \\
\hline Peripheral vascular disease & $2(3.1)$ \\
\hline Smoking history, n (\%) & $48(75)$ \\
\hline History of alcohol abuse, n (\%) & $10(15.6)$ \\
\hline \multicolumn{2}{|l|}{ Reasons for esophageal procedure, $\mathrm{n}(\%)$} \\
\hline Malignant neoplasm & $37(57.8)$ \\
\hline Esophageal perforation & $14(21.9)$ \\
\hline Achalasia & $3(4.7)$ \\
\hline Stricture & $3(4.7)$ \\
\hline Other & 7 (10.9) \\
\hline \multicolumn{2}{|l|}{ Esophageal procedure performed, n (\%) } \\
\hline Total esophagectomy & $6(9.4)$ \\
\hline Partial esophagectomy & $44(68.8)$ \\
\hline Stent & $1(1.6)$ \\
\hline Primary repair with muscle flap & $8(12.5)$ \\
\hline Primary repair without muscle flap & $4(6.3)$ \\
\hline Drain placement & $1(1.6)$ \\
\hline \multicolumn{2}{|l|}{ Part of esophagus involved, $\mathrm{n}(\%)$} \\
\hline Upper & $1(1.6)$ \\
\hline Middle & $5(7.8)$ \\
\hline Lower & $56(87.5)$ \\
\hline \multicolumn{2}{|l|}{ Imaging performed, n (\%) } \\
\hline Grape juice and other imaging modality & $1(1.6)$ \\
\hline Grape juice and Barium swallow & $63(98.4)$ \\
\hline
\end{tabular}

as this is considered to be the reference standard. For all patients in this study, it was typical that they received the barium swallow study on the same post-operative day as their grape juice study.

\section{Exposure and outcome variables}

Data collected included basic patient demographics, medical and surgical history, indication for surgery, treatment strategy, operative findings, imaging results, and grape juice test results. All patients underwent a barium swallow study, CT imaging or upper endoscopy, as well as a grape juice study on POD 5 or greater. Purple grape juice observed in the tube thoracostomy drainage system was identified as a positive esophageal leak. Positive results were recorded in the electronic medical record and identified by the research team. Positive results for esophageal leaks on barium swallow study, CT imaging or endoscopy were recorded from data collection in the electronic medical record without separate interpretation performed at the time of research data collection. The primary outcome of interest was esophageal anastomotic leaks detected with grape juice swallow study. Secondary outcomes of interest included hospital length of stay (LOS), ventilatory support hours, readmission rates, post-operative complications, and mortality. Sensitive and specificity data for barium swallow studies were not reported in this study. This is because barium swallow studies were considered the gold standard and there was no control group for adequate comparison.

\section{Statistical analysis}

Grape juice efficacy, standard sensitivity and specificity calculations were performed using Microsoft Excel (Redmond, Washington). Chi-squared (Fisher's exact) and Mann-Whitney non-parametric $t$-test were performed using GraphPad Prism (San Diego, CA, USA).

\section{Results}

\section{Patient characteristics}

Sixty-four participants were included in the study period ( $25 \%$ female, $88 \%$ white, and median age of 62 years old), Table 1. More than half had a co-morbid history of hypertension $(37,58 \%)$, while a large majority (48, 75\%) were current or former smokers at the time of surgery. Treatment of a malignant neoplasm was the primary 


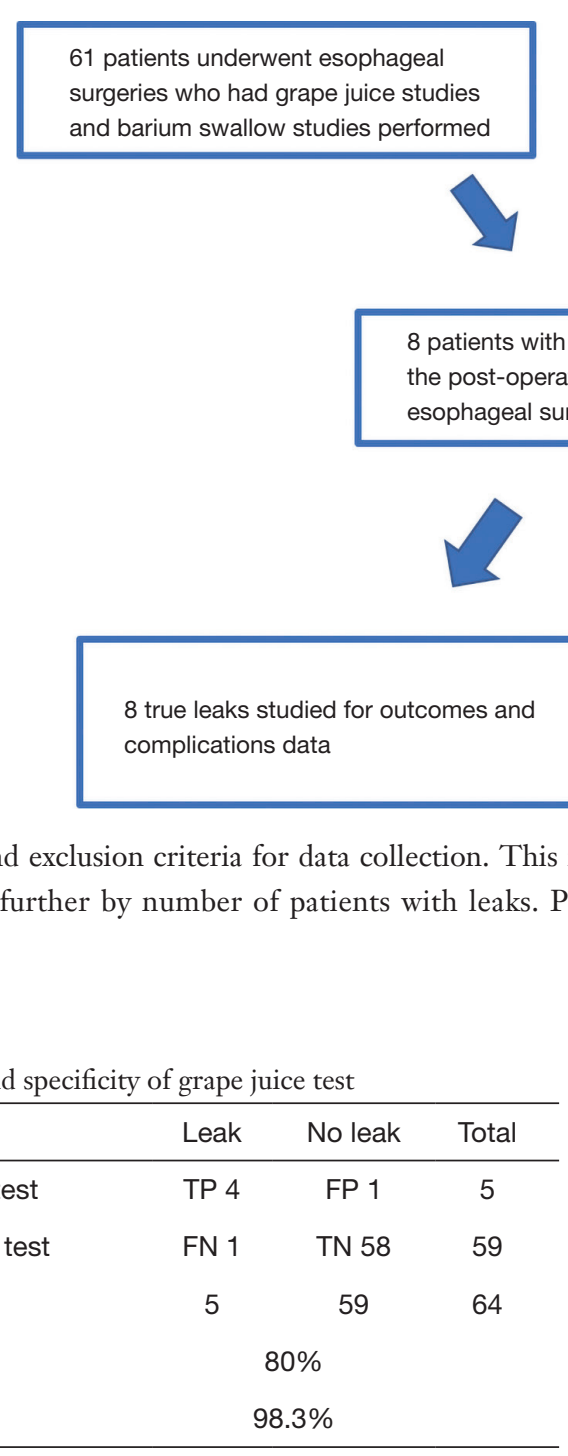

Table 2 Sensitivity and specificity of grape juice test

\begin{tabular}{lccc}
\hline Variable & Leak & No leak & Total \\
\hline Positive grape juice test & TP 4 & FP 1 & 5 \\
Negative grape juice test & FN 1 & TN 58 & 59 \\
Total & 5 & 59 & 64 \\
Sensitivity & \multicolumn{2}{c}{$80 \%$} & \\
Specificity & \multicolumn{2}{c}{$98.3 \%$} \\
\hline
\end{tabular}

reason $(37,58 \%)$ for esophageal operations. Partial esophagectomies of the lower esophagus was the most frequent operation performed (44, 69\%). Sixty-one patients (95\%) had both a barium swallow and grape juice study, while one and two patients had CT imaging and upper endoscopies, respectively.

\section{Sensitivity and specificity}

In total, there were eight $(12.5 \%)$ true post-operative esophageal leaks. However, only five of these true leaks were eligible for sensitivity and specificity calculations due to the timing of the grape juice study being after the leak repair (Figure 1). Of these, four leaks were detected on analysis.
3 patients underwent esophageal surgery who had grape juice study and other imaging modality performed

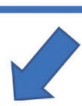

Figure 1 Inclusion and exclusion criteria for data collection. This represents the 64 patients who were included, broken down by imaging modalities, and then further by number of patients with leaks. Patients with leaks were further excluded for sensitivity and specificity

5 true leaks evaluated for sensitivity and specificity data (3 patients excluded because grape juice study was performed after leak repair) grape juice and barium swallow study, representing true positives. Among the 64 participants, there were 59 true negative studies. There was also one false negative and one false positive. Based on standard calculations, the sensitivity and specificity are $80 \%$ and $98.3 \%$, respectively (Table 2).

The false negative patient had an iatrogenic esophageal perforation. An outside hospital chest CT showed pneumomediastinum and a large fluid collection representing a contained leak in the esophagus. Their esophageal perforation was treated with a primary repair with an intercostal muscle flap closure and placement of a 32 French chest tube. On POD6, the false negative patient had a barium swallow study which showed persistence of a tiny, contained leak. This patient was treated with medical management and continued on total parenteral nutrition and received nothing by mouth. They were clinically stable despite the tiny leak and on POD9, a purple grape juice test was negative. Their diet was appropriately advanced and was discharged on POD11. We postulate that the false negative result was likely due to the leak being too small and contained, which did not allow for detection of purple grape juice in the tube thoracostomy system. It does not appear that malposition of the chest tube, chest tube size, or surgical procedure had any impact on this result when compared to the other true positive leaks. 
Table 3 Outcomes among patients with and without esophageal leaks

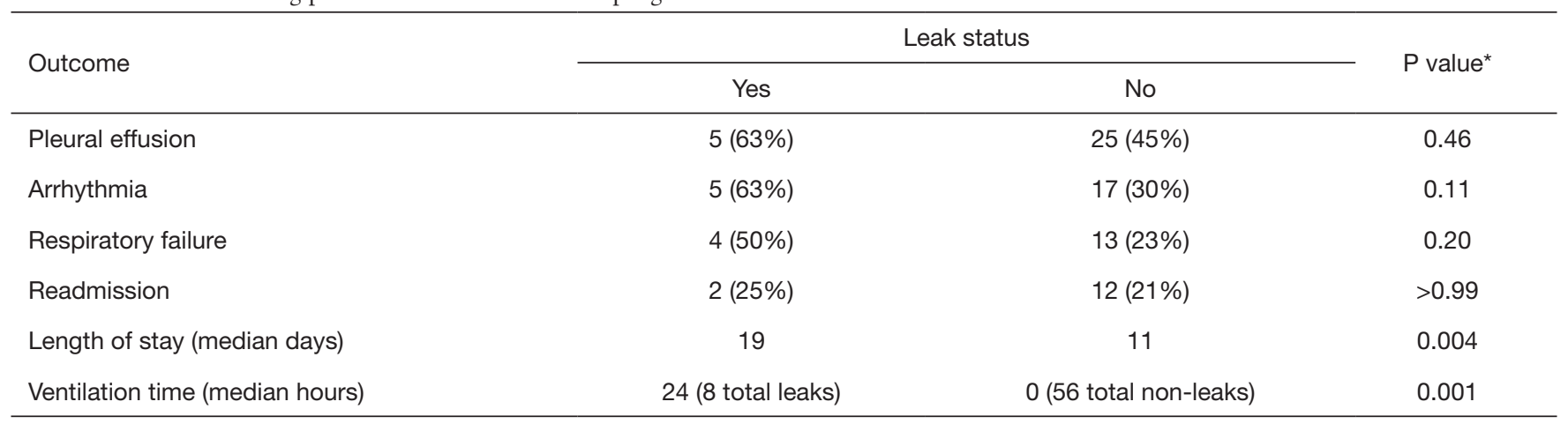

${ }^{*}, \mathrm{P}<0.05$ is considered statistically significant.

The other confirmed leaks consisted of three malignant neoplasms and one esophageal perforation. The initial operations of the neoplasms included two partial esophagectomies and one total esophagectomy, while the perforation was treated by primary repair with muscle flap closure. The leaks were confirmed in the range of POD3-10. Two of these leaks were treated with medical management which includes clear liquid diet, J-tube feedings, and maintenance of the tube thoracostomy until leak resolution. The other two confirmed leaks were treated with esophageal stent placement. Their hospital courses were complicated by pneumothorax, pneumonia, empyema, small bowel obstruction, new-onset arrhythmias, and respiratory failure requiring prolonged ventilation and in one case, tracheostomy. One of these patients also required readmission for sepsis. Similarly to the false negative, they received 28-32 French chest tubes which stayed for on average 9.75 days. These patients were all discharged following leak resolution, chest tube removal, toleration of clear liquid diet, and appropriately management of postoperative complications.

\section{Esophageal leak outcomes}

Within the leak population, four patients $(50 \%)$ had initial esophageal operations as an elective procedure for malignant neoplasms and one case of a mediastinal abscess causing dysphagia. All four elective cases were Ivor-Lewis esophagectomies, with three being partial resections and one requiring total resection. The other leaks were preceded by esophageal repairs for emergency esophageal perforations. The surgical techniques for these included three primary repairs with a muscle flap and one without a flap. It is noteworthy that the sole mortality within the overall group of 64 patients was in a non-leak patient who suffered an iatrogenic esophageal perforation.

Patients who underwent esophagectomies or esophageal repairs and had post-operative anastomotic leaks were compared to those without evidence of a leak. These comparisons showed that patients who suffered from an anastomotic leak during their post-operative period had a significant greater LOS (median days: 19 days, $\mathrm{P}=0.004$ ) and total hours on ventilation (median: 24 hours, $\mathrm{P}=0.001$ ) (Table 3). Other measures that showed no significant differences were rates of readmission and post-operative complications including pleural effusions, arrhythmias, and respiratory failure (Table 3). There were also no recorded adverse events associated with either grape juice or barium swallow studies.

\section{Discussion}

Esophageal anastomotic leaks are a major post-operative complication following esophagectomies and esophageal repairs. To our knowledge, there have been no specific reports analyzing the sensitivity or specificity of visualization of oral purple grape juice studies in a tube thoracostomy drainage system for the detection of anastomotic leak following esophagectomy or esophageal repair. More broadly, there have been no reports showing the utility of oral juice swallow studies, including other colored beverage variants such as cranberry juice or food-colored water. In our study, there was adequate detection of esophageal leaks using this method when compared to other standard imaging modalities (barium swallow, CT, and endoscopy). The high specificity of $98.3 \%$ suggests the ability to confidently rule out a leak with a negative visualization of grape juice in the thoracostomy drainage system. Although 


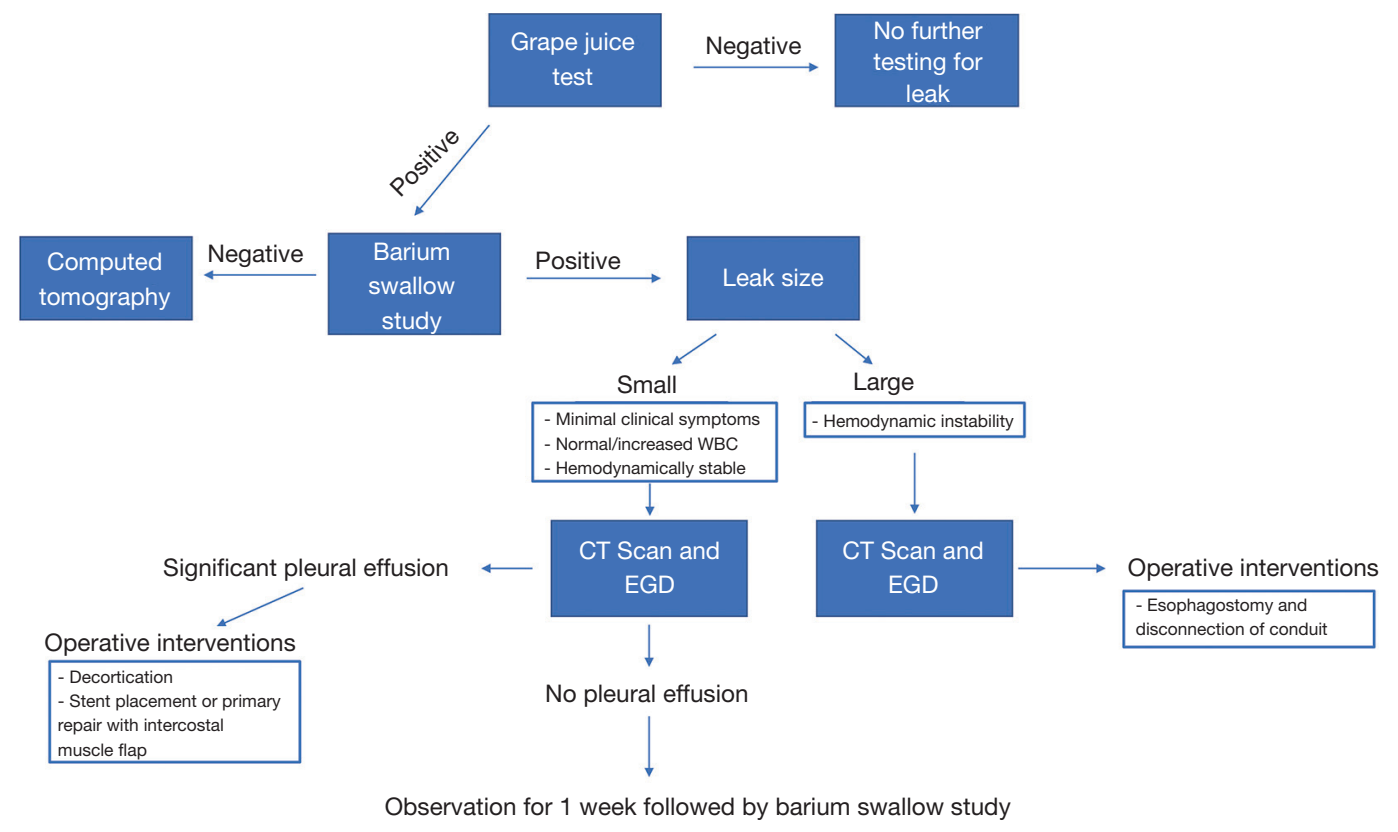

Figure 2 Algorithm for detection and management of esophageal anastomotic leak. This represents the current clinical utility of the grape juice swallow study at our institution and the clinical decision making pathway depending on test results and patient presentation. EGD, esophagogastroduodenoscopy.

the sensitivity is less than the specificity, this suggests its utility as a screening tool rather than a gold standard for diagnosis. For example, a patient on POD 5 who is afebrile and without cough or neck wound drainage would be an ideal candidate for a grape juice test. The absence of purple grape juice in the tube thoracostomy would suggest structural integrity of the esophageal anastomoses. With a negative screen, this eliminates the need to perform a barium swallow study which prevents contrast exposure, lowers potential complications, and reduces financial cost to the patient. At this point with a negative grape juice test (or barium swallow study), a patient could have the chest tube removed and be advanced to a clear liquid diet.

A study from Baker et al. found similar approaches for pleural drain amylase, where it was used as a screening tool in conjunction with white blood cell counts to determine whether the likelihood of a leak was increased and an indication for CT imaging (15). However, if grape juice was present in tube thoracostomy, this would suggest a possible structural defect in the esophageal anastomoses. This positive test would indicate the use of other diagnostic testing such as CT or endoscopy to better characterize the location, size, and etiology of the leak and would effectively eliminate the use for barium contrast studies (Figure 2).

Based on our study, esophagogastric anastomotic leak rates for our institution $(12.5 \%)$ is within the range compared to other facilities who investigated the use of other leak imaging modalities (6-21\%) against barium swallow studies (1,4-10,15-18). The majority of these studies were similar to ours as they were retrospective in nature and focused on comparing barium swallows as the gold standard to another modality they hypothesized to be superior. None of these studies firmly asserted that their method, such as CT imaging, endoscopy, C-reactive protein (CRP), or pleural drain amylase test, could adequately serve as a replacement for routine diagnostic screening of anastomotic leaks. These diagnostic tools have their highest clinical utility when patients are symptomatic or there are clinical indications that a leak may be present, rather than performed for routine use in asymptomatic patients. While we recognize there are many techniques for esophageal leak detection, we chose grape juice as it presented as a readily accessible and inexpensive tool in the hospital setting with minimal risk for complications. A concern regarding the use of grape juice, as well as the other techniques to evaluate for leak is the difficulty with identifying small, minor, or contained leaks.

This current study aimed to investigate if grape juice could provide utility in detection of an anastomotic leak even prior to clinical suspicion. Patient outcomes in current 
literature have reported mortality rates following esophageal leak between $10-50 \%(2,6)$. In this study, we focused on patient and disease-centered outcomes such as LOS, hours on mechanical ventilation, and other post-operative complication rates. Median LOS and time on ventilators were significantly greater for patients who suffered an anastomotic leak post-operatively. Roh et al. reported similar statistically significant differences in median LOS, while Schaible et al. described greater LOS on the basis of clinical significance $(4,6)$. This indicates that patients with postoperative esophageal anastomotic leaks were more acutely ill and at greater risk for post-operative complications.

Our institution, as well as others, utilizes routine barium swallow studies as their standard of care for leak detection following esophagectomy and esophageal repairs. Other studies have shown CT imaging and post-operative endoscopy as increasingly popular methods of detection, due to the poor sensitivity and specificity found using contrast mediated swallow studies. However, these have yet to become widely adopted due to concerns regarding reliable differences in sensitivity and iatrogenic injury for CT imaging and endoscopy respectively $(6,14)$.

Currently at our institution, thoracic surgeons utilize barium swallow studies on POD 5 or greater, in addition to grape juice studies for routine detection of anastomotic leaks. There has not been a complete shift to using grape juice studies over barium swallow studies in the routine detection of esophageal leaks due to the sample size limitations. Other surgeons throughout our institution such as surgical oncologists have also shown interest in utilizing grape juice studies for leak detection, however they continue to perform barium swallow studies and drain amylase studies based on physician preference. With a widespread adoption of grape juice studies at our institution, this could potentially be a route to increase our study power and further investigate the utility and strength of grape juice in the routine detection of esophageal anastomotic leaks.

\section{Limitations}

There are several limitations to this study. First, this was a retrospective single-center study with a small sample size. Due to the limited sample size, our study was underpowered to detect small differences between leak versus non-leak groups for post-operative complications. These drawbacks limit the power of grape juice as a reliable screening at this point. With the use of the grape juice test becoming more widely adopted at our institution, we hope to include more patients in the future to further bolster our data and strengthen this investigation.

Another limitation is our lack of long-term data after 30 days for these patients. We did not measure re-admission or mortality beyond 30 days, and whether patients with leaks had increased morbidity in comparison to their nonleak counterparts. A randomized, prospective trial would show differences in long-term morbidity and mortality for patients with esophageal anastomotic leaks, and provide statistical validation that grape juice studies are more reliable than barium swallow studies.

\section{Conclusions}

Our findings suggest that the purple grape juice may be an effective initial screening tool for the detection of esophageal anastomotic leaks in patients with tube thoracostomies. Grape juice is a cheaper option with potentially less associated morbidities than other diagnostic modalities. However, a positive grape juice test will still require another diagnostic technique to better characterize the leak for further medical or surgical management. Further research is needed to justify the increased use of grape juice in patients who undergo esophageal operations and may be best tested in a multi-institutional setting with a prospective approach. This would also include exploring the difference in cost between barium swallow studies and the grape juice test.

\section{Acknowledgments}

This study was presented during The 16th Annual American Surgical Congress (virtual experience, oral presentation). Funding: None.

\section{Footnote}

Reporting Checklist: The authors have completed the STARD reporting checklist. Available at https://dx.doi. org/10.21037/jtd-21-1185

Data Sharing Statement: Available at https://dx.doi. org/10.21037/jtd-21-1185

Peer Review File: Available at https://dx.doi.org/10.21037/ jtd-21-1185

Conflicts of Interest: All authors have completed the ICMJE 
uniform disclosure form (available at https://dx.doi. org/10.21037/jtd-21-1185). The authors have no conflicts of interest to declare.

Ethical Statement: The authors are accountable for all aspects of the work in ensuring that questions related to the accuracy or integrity of any part of the work are appropriately investigated and resolved. The study was conducted in accordance with the Declaration of Helsinki (as revised in 2013). The study was approved by institutional review board committee (IRB\#: 20-0830) and individualized consent for this retrospective analysis was waived.

Open Access Statement: This is an Open Access article distributed in accordance with the Creative Commons Attribution-NonCommercial-NoDerivs 4.0 International License (CC BY-NC-ND 4.0), which permits the noncommercial replication and distribution of the article with the strict proviso that no changes or edits are made and the original work is properly cited (including links to both the formal publication through the relevant DOI and the license). See: https://creativecommons.org/licenses/by-nc-nd/4.0/.

\section{References}

1. Page RD, Asmat A, McShane J, et al. Routine endoscopy to detect anastomotic leakage after esophagectomy. Ann Thorac Surg 2013;95:292-8.

2. Persson S, Rouvelas I, Irino T, et al. Outcomes following the main treatment options in patients with a leaking esophagus: a systematic literature review. Dis Esophagus 2017;30:1-10.

3. Griffin SM, Lamb PJ, Shenfine J, et al. Spontaneous rupture of the oesophagus. Br J Surg 2008;95:1115-20.

4. Roh S, Iannettoni MD, Keech JC, et al. Role of Barium Swallow in Diagnosing Clinically Significant Anastomotic Leak following Esophagectomy. Korean J Thorac Cardiovasc Surg 2016;49:99-106.

5. Solomon DG, Sasaki CT, Salem RR. An evaluation of the routine use of contrast radiography as a screening test for cervical anastomotic integrity after esophagectomy. Am J Surg 2012;203:467-71.

6. Schaible A, Sauer P, Hartwig W, et al. Radiologic versus endoscopic evaluation of the conduit after esophageal

Cite this article as: Malfitano MJ, Bui JT, Swier RM, Haithcock BE. The use of grape juice in the detection of esophageal leaks. J Thorac Dis 2021;13(11):6323-6330. doi: 10.21037/jtd-21-1185 resection: a prospective, blinded, intraindividually controlled diagnostic study. Surg Endosc 2014;28:2078-85.

7. Boone J, Rinkes IB, van Leeuwen M, et al. Diagnostic value of routine aqueous contrast swallow examination after oesophagectomy for detecting leakage of the cervical oesophagogastric anastomosis. ANZ J Surg 2008;78:784-90.

8. Strauss C, Mal F, Perniceni T, et al. Computed tomography versus water-soluble contrast swallow in the detection of intrathoracic anastomotic leak complicating esophagogastrectomy (Ivor Lewis): a prospective study in 97 patients. Ann Surg 2010;251:647-51.

9. Upponi S, Ganeshan A, D'Costa H, et al. Radiological detection of post-oesophagectomy anastomotic leak - a comparison between multidetector CT and fluoroscopy. Br J Radiol 2008;81:545-8.

10. Tirnaksiz MB, Deschamps C, Allen MS, et al. Effectiveness of screening aqueous contrast swallow in detecting clinically significant anastomotic leaks after esophagectomy. Eur Surg Res 2005;37:123-8.

11. Chen A, Tafti D, Tuma F. Barium Swallow 2021.

12. Agha FP, Orringer MB, Amendola MA. Gastric interposition following transhiatal esophagectomy: radiographic evaluation. Gastrointest Radiol 1985;10:17-24.

13. Tanomkiat W, Galassi W. Barium sulfate as contrast medium for evaluation of postoperative anastomotic leaks. Acta Radiol 2000;41:482-5.

14. Hogan BA, Winter DC, Broe D, et al. Prospective trial comparing contrast swallow, computed tomography and endoscopy to identify anastomotic leak following oesophagogastric surgery. Surg Endosc 2008;22:767-71.

15. Baker EH, Hill JS, Reames MK, et al. Drain amylase aids detection of anastomotic leak after esophagectomy. J Gastrointest Oncol 2016;7:181-8.

16. Miller DL, Helms GA, Mayfield WR. Evaluation of Esophageal Anastomotic Integrity With Serial Pleural Amylase Levels. Ann Thorac Surg 2018;105:200-6.

17. Honing J, Pultrum BB, van der Jagt EJ, et al. Routine or on demand radiological contrast examination in the diagnosis of anastomotic leakage after esophagectomy. J Surg Oncol 2009;100:699-702.

18. Gordon AC, Cross AJ, Foo EW, et al. C-reactive protein is a useful negative predictor of anastomotic leak in oesophago-gastric resection. ANZ J Surg 2018;88:223-7. 